\title{
Efficacy of intravenous acetaminophen in multimodal management for pain relief following total knee arthroplasty: a meta- analysis
}

\author{
Song-bo Shi, Xing-bo Wang, Jian-min Song, Shi-fang Guo, Zhi-xin Chen and Yin Wang*
}

\begin{abstract}
Background: The efficacy of intravenous acetaminophen in multimodal pain management in patients undergoing total knee arthroplasty (TKA) is controversial. The purpose of this meta-analysis was to compare the efficacy of intravenous acetaminophen versus placebo in TKA.

Methods: Randomized controlled trials (RCTs) or retrospective cohort studies (RCSs) concerning related topics were retrieved from PubMed (1996-June 2018), Embase (1980-June 2018), and the Cochrane Library (CENTRAL June 2018). Any studies comparing intravenous acetaminophen with a placebo were included in this meta-analysis. Meta-analysis results were collected and analyzed by Stata 12.0. Subgroup analysis was performed according to the general characteristics of the patients.

Results: In total, the patients from six studies met the inclusion criteria. Our meta-analysis results indicated that compared with a control group, intravenous acetaminophen was associated with reductions in total morphine consumption and visual analogue scale (VAS) score at postoperative day (POD) 3. However, there was no significant difference in morphine consumption at POD 1 or in VAS at POD 1 or POD 2. Moreover, there was no significant difference in the length of hospital stay.

Conclusions: Based on our results, intravenous acetaminophen in multimodal management has shown better efficacy in pain relief at POD 3 and has morphine-sparing effects. High-quality studies with more patients are needed in the future.
\end{abstract}

Keywords: Acetaminophen, Pain control, Total knee arthroplasty, Meta-analysis

\section{Background}

Total knee arthroplasty (TKA) is being widely used for end-stage osteoarthritis (OA) or rheumatoid arthritis (RA) [1]. However, over $80 \%$ of TKA patients experience severe to moderate postoperative pain [2]. Inadequate pain management may result in dissatisfaction, complications, stunted postoperative functional recovery, and longer hospital stays [3, 4]. Conventionally, multimodal pain management is widely recommended and accepted $[5,6]$. Multimodal pain management usually includes two or more analgesics, such as opioids, nonsteroidal

* Correspondence: 246771930@qq.com

Orthopaedics Department, Gansu Provincial Hospital, Lanzhou 730000, Gansu, China anti-inflammatory medications, steroid hormones, and epinephrine [7]. It has been reported that rebounding pain in patients treated with multimodal pain management after $24 \mathrm{~h}$ postoperatively remains a real problem for surgeons $[8,9]$. Furthermore, the use of opioids is frequently associated with some side effects, including gastrointestinal symptoms, autonomic nervous system symptoms, and central nervous system symptoms, among others $[10,11]$. Thus, adjunctive pain management medication is needed.

Recently, combination therapy with intravenous (IV) acetaminophen has been used to reduce postoperative pain, and opioid use across a variety of surgical procedures has also been applied to the TKA [12-14]. Kelly et al. [15] 
drew the conclusion that IV acetaminophen did not significantly decrease postoperative opioid use in patients who underwent surgical knee procedures. Studies conducted by Blank et al. [16] and Nwagbologu et al. [17] presented similar conclusions. However, O'Neal et al. [18] reported that neither IV nor oral acetaminophen provided better analgesia in patients undergoing TKA. Similar results were reported by subsequent studies [19]. Thus, conclusions concerning the use of IV acetaminophen in reducing postoperative pain and opioid consumption have been inconsistent. Several studies have reported that IV acetaminophen has a beneficial role in reducing pain intensity and morphine consumption after TKA [19, 20]. However, some other studies suggest that the use of acetaminophen in multimodal pain management does not result in improved safety or reduced opioid utilization in hip or knee arthroplasty [17, 21].

Therefore, it is necessary to investigate whether IV acetaminophen as an adjunctive pain management medication provides better analgesic effects, as well as whether it reduces opioid consumption in patients after TKA. The purpose of the current meta-analysis was to compare results concerning the efficacy of IV acetaminophen for pain control in patients undergoing TKA.

\section{Methods \\ Search strategy}

We manually searched randomized controlled trials (RCTs), retrospective cohort studies (RCSs), and cohort studies through PubMed (1996-June 2018), Embase (1980-June 2018), and the Cochrane Library (CENTRAL, June 2018). We also searched trials from related references and reviews. The key words and $\mathrm{MeSH}$ terms were "total knee arthroplasty," "total knee replacement," "TKA," "TKR," "Arthroplasty, Replacement, Knee" $[\mathrm{MeSH}]$, and "acetaminophen." These key words or MeSH terms were combined using the Boolean operators "AND" or "OR." The search results are presented in Fig. 1.

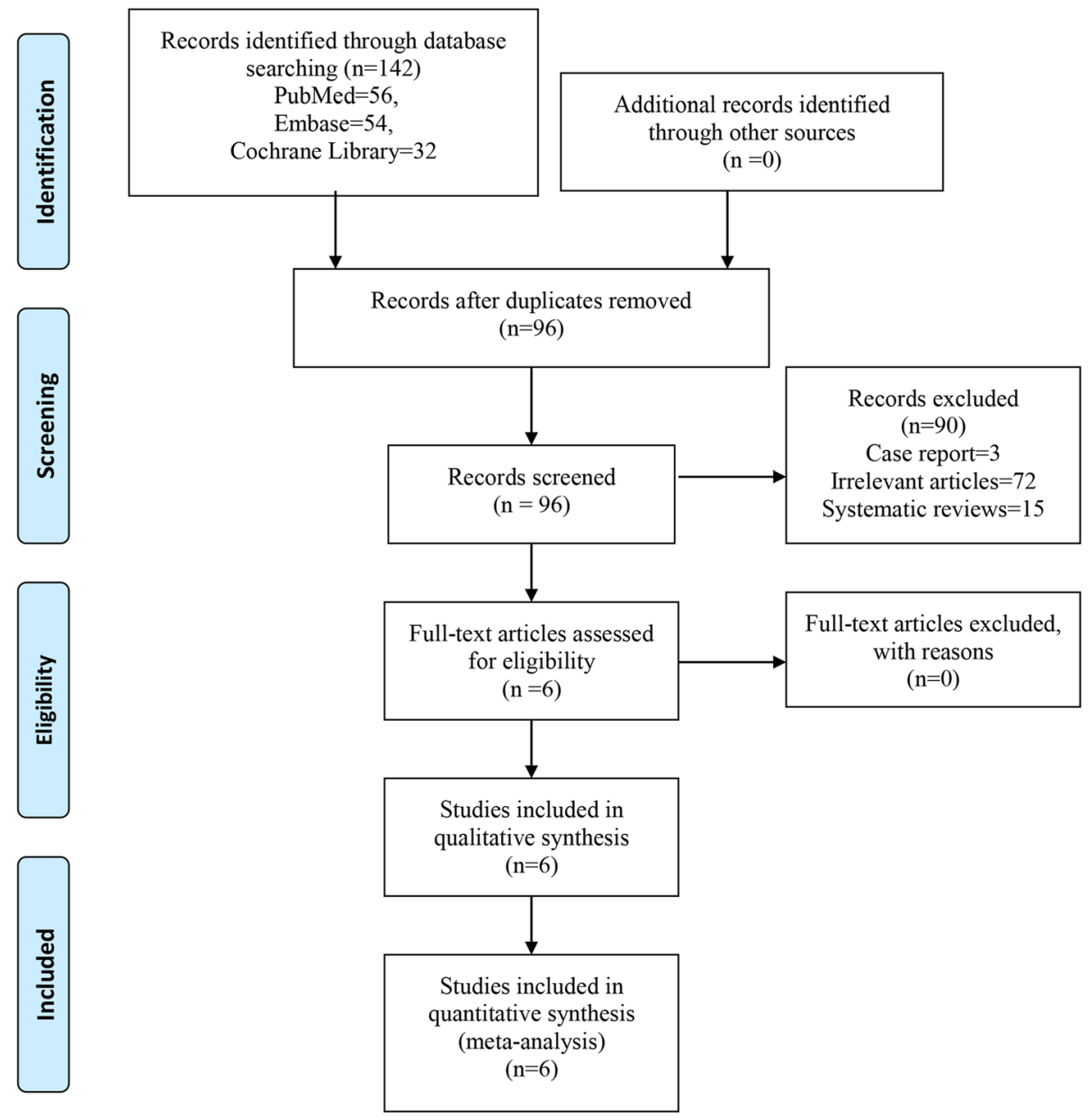

Fig. 1 Flow of trials through the meta-analysis 
Table 1 General characteristic of the included studies

\begin{tabular}{llllllll}
\hline Author & Country & Age (year) & Study & Dose of acetaminophen & Control & Follow-up & Anesthesia \\
\hline Kelly [15] & America & $63.9 / 65.3$ & RCS & $1000 \mathrm{mg} /$ day & Placebo & NS & NS \\
Nwagbologu [17] & Mexico & $61 / 63.9$ & RCS & $1000 \mathrm{mg} /$ day & Placebo & 3 days & NS \\
O'Neal [18] & America & $68 / 70$ & RCT & $1000 \mathrm{mg} /$ day & Placebo & NS & SA \\
Murata-Ooiwa [19] & Japan & $73.6 / 75.3$ & RCT & $1000 \mathrm{mg} /$ day & Placebo & 3 days & SA \\
Ciummo [23] & America & NS & RCS & NS & Placebo & NS & NS \\
Huang [21] & America & $71.3 / 71.6$ & RCS & $4000 \mathrm{mg} /$ day & Placebo & 3 days & SA/GA
\end{tabular}

$R C T$ randomized controlled trials, $R C S$ retrospective controlled studies, NS not stated, SA spinal anesthesia, GA general anesthesia

\section{Inclusion criteria}

Studies were included in our meta-analysis provided that they satisfied the condition of meeting the PICOS (patients, intervention, comparator, outcomes, and study design) study quality assurance guidelines. Other inclusion criteria included the following: (1) Patients had undergone TKA. (2) The intervention was intravenous acetaminophen. (3) The comparator was non-intravenous administration of acetaminophen or placebo. (4) The outcomes included morphine equivalent consumption at POD 1, total morphine equivalent consumption, VAS score at 24, 48, and $72 \mathrm{~h}$ and length of hospital stay.

\section{Data extraction}

Two reviewers extracted available data from the included studies independently. Extracted data included first author, publication data, participants, age, gender, body mass index, and study design. The primary outcome of our meta-analysis consisted of morphine equivalent consumption at POD 1, total morphine consumption, and VAS score at 24, 48, and $72 \mathrm{~h}$

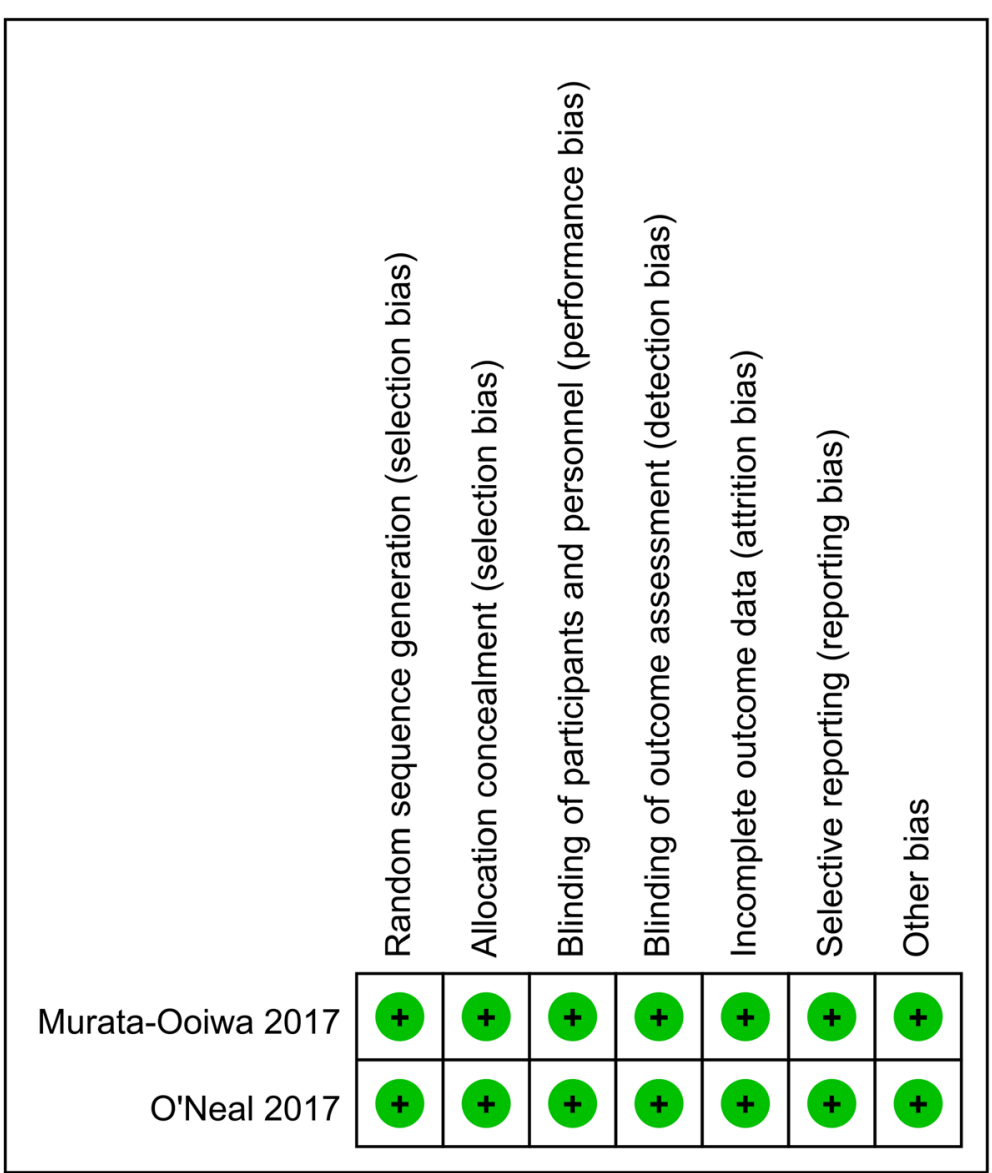

Fig. 2 Risk of bias summary of the RCTs 


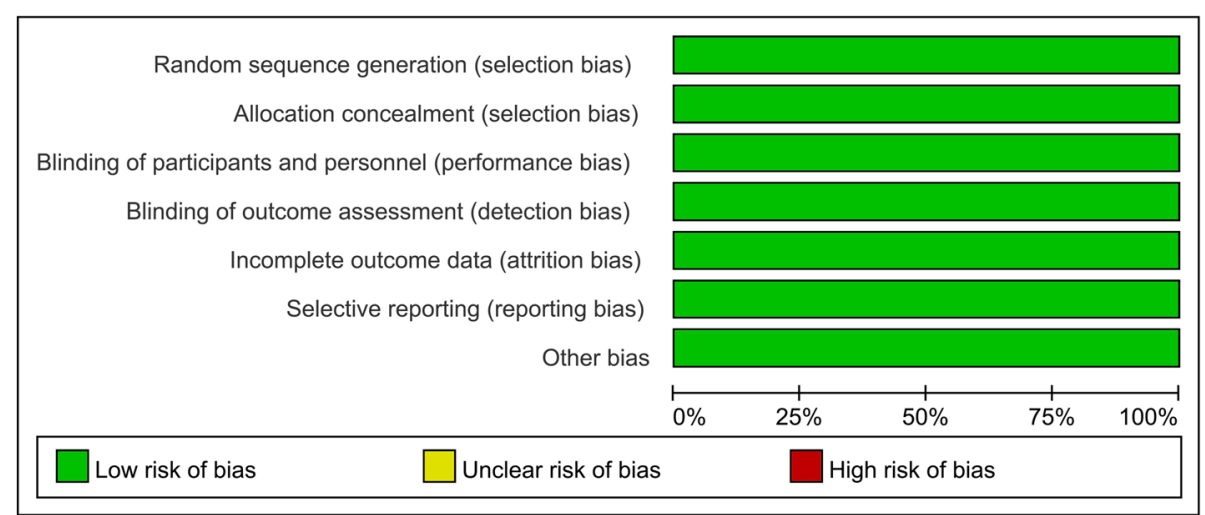

Fig. 3 Risk of bias graph of the RCTs

postoperatively. Secondary outcomes consisted of length of hospital stay. We tried emailing the corresponding authors of the studies that used graphical data or had incomplete data. Any disagreement between the two reviewers was resolved by a third reviewer.

\section{Quality assessment}

Quality assessment for RCTs was performed according to the Cochrane Handbook for Systematic Reviews of Interventions. Two authors independently evaluated the risk of bias of the included RCTs based on the following items: random sequence generation, allocation concealment, blinding, incomplete outcome data, selective reporting, and other sources of bias. For non-RCTs, we used the Newcastle-Ottawa scale to evaluate the risk of bias [22]. We considered a study to be of high quality for non-RCTs when a study achieved a score on the Newcastle-Ottawa scale of more than six points.

\section{Statistical analysis}

Stata 12.0 was applied for our meta-analysis. For continuous outcomes, mean difference (MD) with a $95 \%$ confidence interval $(\mathrm{CI})$ was used to weigh the effect intervals. We judged the statistical heterogeneity by the $P$ value derived using the standard chi-square test. Values of $I^{2}>50 \%$ were thought to have significant heterogeneity of the outcomes, and a random-effect model was applied for assessment; for others, such as for extracted data, a fixed-effect model was used. We performed subgroup analysis by omitting studies in turn. Subgroup analysis was done according to the study type, anesthesia, allocation concealment, and dose of acetaminophen.

\section{Results}

Search results and general characteristics

A total of 142 relevant studies were identified by our search strategies. After duplicates were removed, there were 96 studies left to review. After reading the title and abstract, 90 studies were excluded. Finally, 6 studies [15, 17-19, 21, 23] were included in our meta-analysis after full-text reading. Among them, there were 2 RCTs [18, 19] and 4 RCSs [15, 17, 21, 23]. General characteristics of the included RCTs can be seen in Table 1. All of the studies were published in 2014. The ages of the TKA patients ranged from 61 to 75.3 years. Four studies [15, 17-19] administered acetaminophen at a dose of $1000 \mathrm{mg} /$ day, and one study administered acetaminophen at a dose of $4000 \mathrm{mg} /$ day [21].

\section{Quality assessment of the included studies}

The risk of bias summary and the risk of bias graph for the RCTs can be seen in Figs. 2 and 3, respectively. The two RCTs were both determined to be of high quality. The quality assessments of the non-RCTs can be seen in Table 2. Total scores of NOS ranged from 6 to 8 .

\section{Meta-analysis results \\ Total morphine equivalent consumption}

Four studies, having a total of 398 patients, reported equivalent total morphine consumptions. Compared with the control group, the IV acetaminophen group

Table 2 Newcastle-Ottawa scale for the non-RCTs

\begin{tabular}{lllll}
\hline Author & Selection & Comparability & Outcomes & Total score \\
\hline Kelly [15] & $* * *$ & $* *$ & $* *$ & 7 \\
Nwagbologu [17] & $* *$ & $* *$ & $* *$ & 6 \\
Ciummo [23] & $* * *$ & $* *$ & $* * *$ & 8 \\
Huang [21] & $* *$ & $* *$ & $* *$ & 6
\end{tabular}

* represent 1 score 


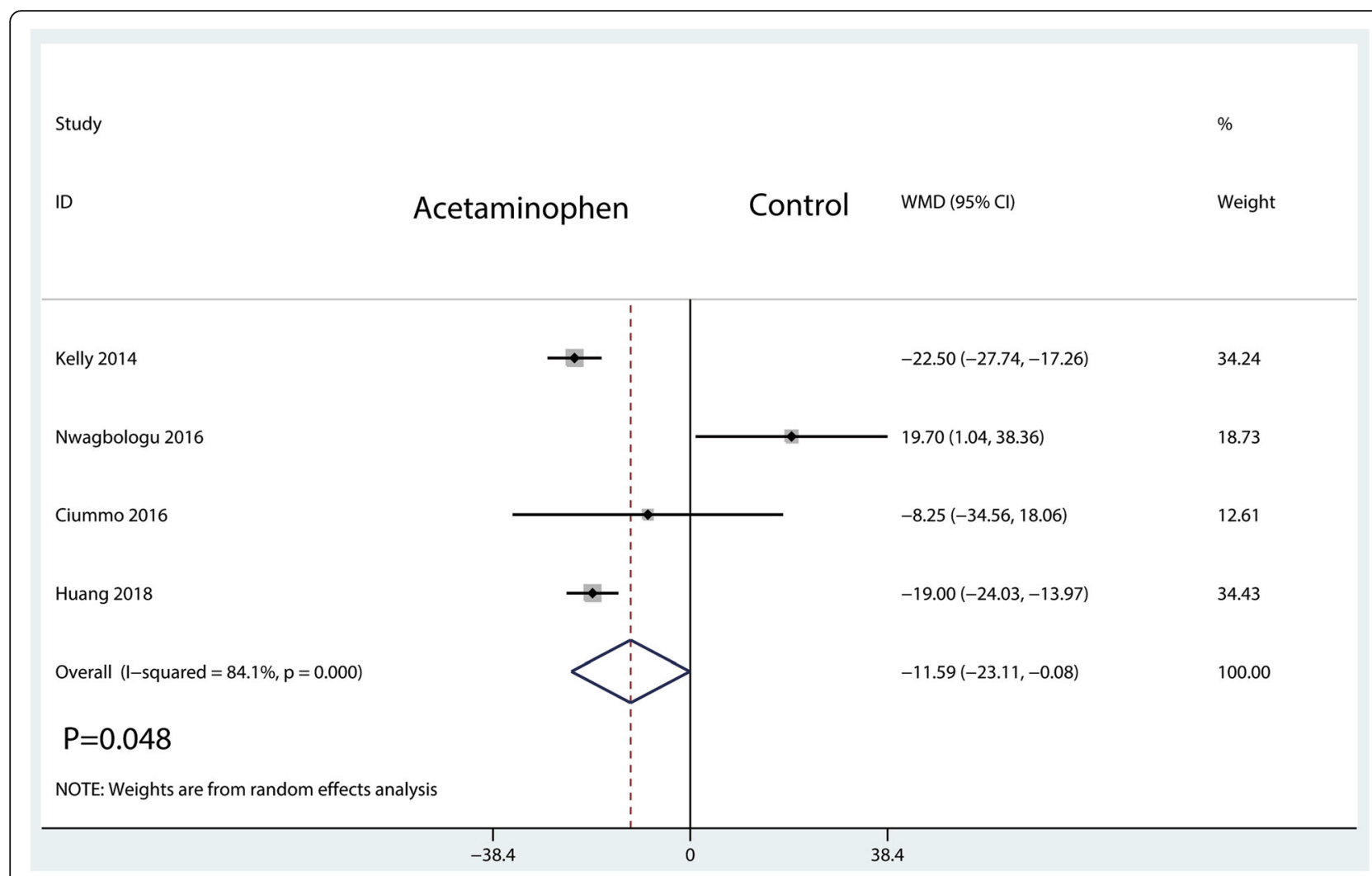

Fig. 4 Forest plots of the included studies comparing the total morphine equivalent consumption

Study

ID
Acetaminophen
Control

WMD $(95 \% \mathrm{Cl})$
$\%$

Weight
Nwagbologu 2016

O'Neal 2017

Overall (I-squared $=41.5 \%, p=0.191)$

\section{$P=0.444$}

NOTE: Weights are from random effects analysis

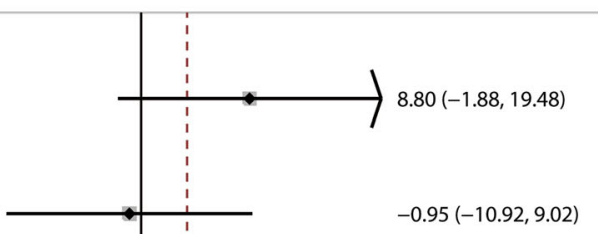

$3.73(-5.82,13.28)$
48.00

52.00

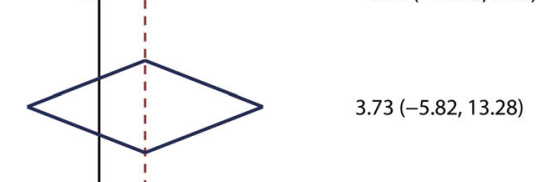

Fig. 5 Forest plots of the included studies comparing the morphine equivalent consumption at POD 1 
was associated with a reduction in total morphine consumption of approximately $11.59 \mathrm{mg}$ (WMD = - 11.59; 95\%CI, [- 23.11, -0.08]; $P=0.048 ; I^{2}=84.1 \%$, Fig. 4).

\section{Morphine equivalent consumption at POD 1}

Data from 2 studies, including 264 patients, reported equivalent morphine consumption at POD 1 . There were no significant differences between the IV acetaminophen group and control group in terms of morphine consumption at POD 1 (WMD $=3.73 ; 95 \% \mathrm{CI}, \quad[-5.82$, 13.28]; $P=0.444 ; I^{2}=41.5 \%$, Fig. 5).

\section{Visual analogue scale at POD 1}

The visual analogue scale score at POD 1 was measured in 4 studies, having a total of 216 patients. We did not find any significant difference between the IV acetaminophen and control groups (WMD $=-4.24 ; 95 \% \mathrm{CI}$, $[-20.24,11.75] ; P=0.603 ; I^{2}=95.2 \%$, Fig. 6$)$.

\section{Visual analogue scale at POD 2}

The visual analogue scale score at POD 2 was measured in 3 studies, having a total of 216 patients. There was no significant difference between IV acetaminophen and control groups in terms of the VAS score at POD 2 (WMD = - 0.26; 95\%CI, [-0.57, 0.05]; $P=0.105 ; I^{2}=0.0 \%$, Fig. 7).

\section{Visual analogue scale at $P O D 3$}

The visual analogue scale score at POD 3 was reported in 4 studies, having a total of 331 patients. Compared with the control group, the IV acetaminophen group was associated with a reduction in VAS score at POD 3 (WMD $=-0.34 ; \quad 95 \% \mathrm{CI}, \quad[-0.68, \quad-0.01] ; \quad P=0.045$; $I^{2}=0.0 \%$, Fig. 8).

\section{Length of hospital stay}

We extracted length of hospital stay data from 4 studies, involving 398 patients. There were no significant differences between the IV acetaminophen and control groups in terms of the length of hospital stay (WMD $=-0.09$; 95\%CI, [- 0.23, 0.05]; $P=0.226 ; I^{2}=58.1 \%$, Fig. 9).

\section{Sensitivity analysis and subgroup analysis}

The sensitivity analysis can be seen in Additional file 1: Figure S1. The results showed that after omitting each study in turn, the overall effects were in the upper CI limit and lower CI limit.

Subgroup analysis results can be seen in Table 3 . The findings of decreased total morphine consumption were consistent in all subgroup analyses except for the allocation concealment and anesthesia subgroups.

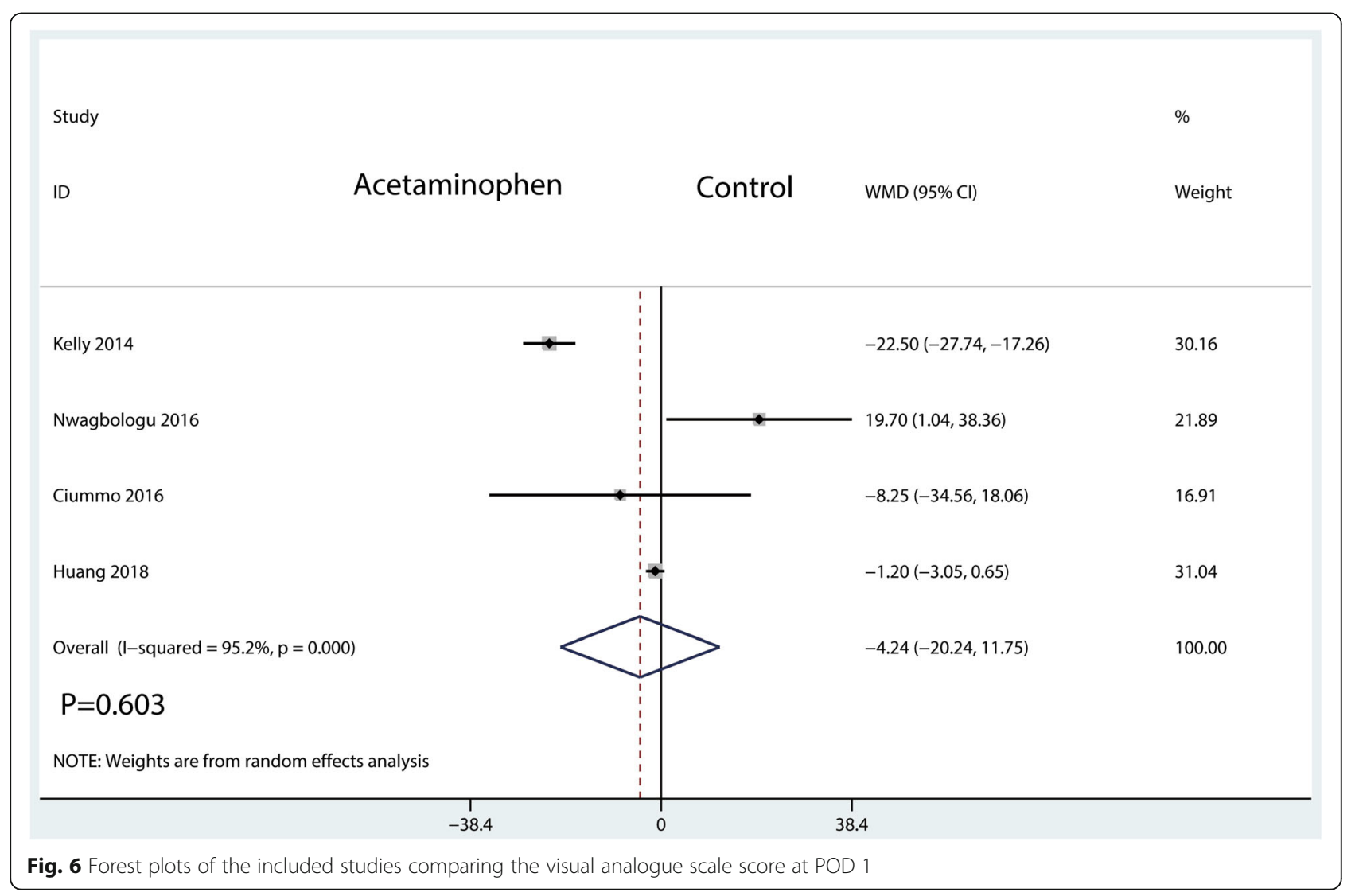




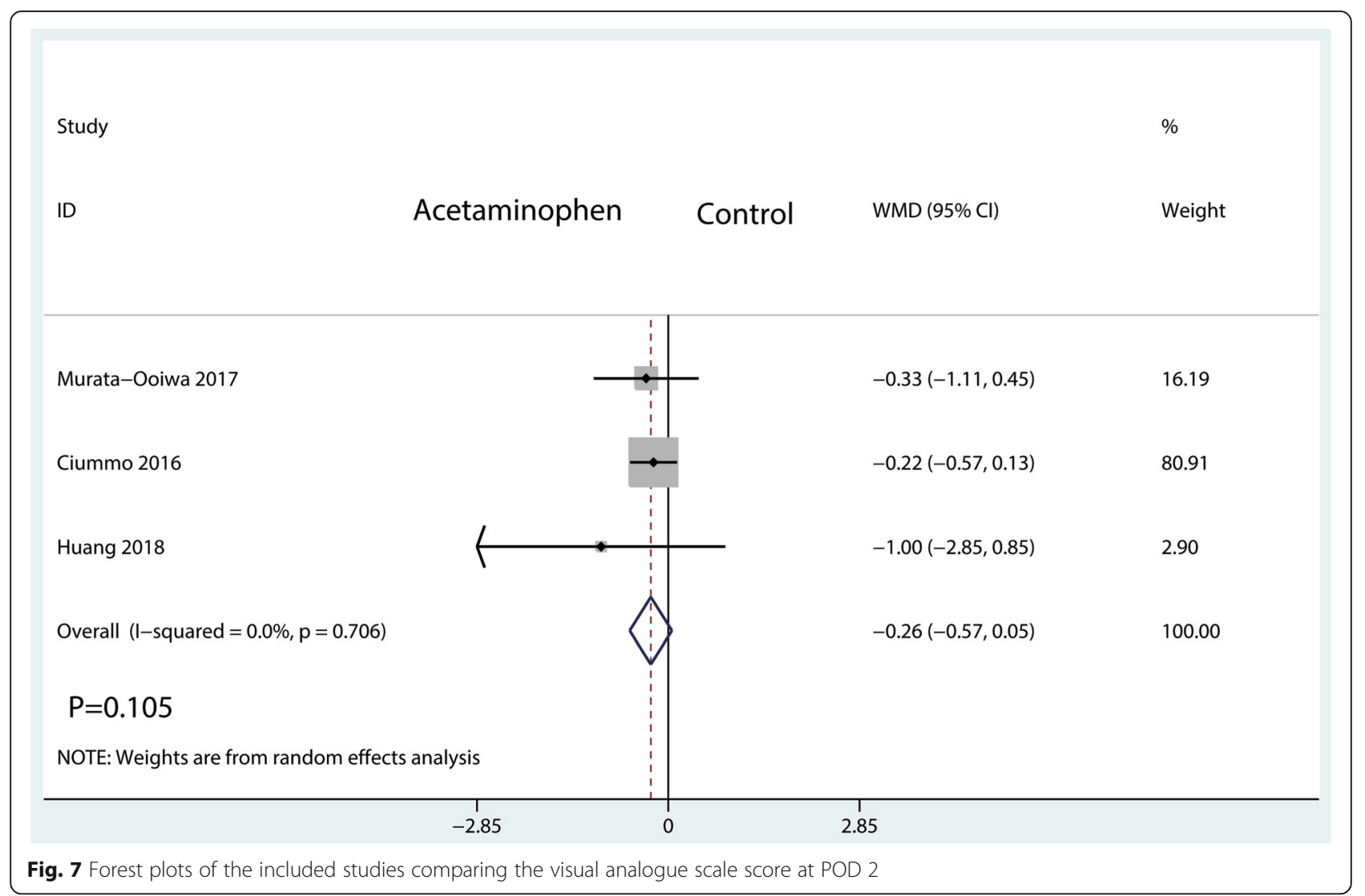

\section{Discussion}

The current meta-analysis indicated that compared with a control group, intravenous acetaminophen was associated with reductions in total morphine consumption and VAS score at POD 3. There was no significant difference in morphine consumption at POD 1 or in VAS score at POD 1 or 2 . Moreover, there was no significant difference in length of hospital stay between the intravenous acetaminophen group and the control group.

Inadequate pain management following TKA may influence the functional recovery, increase opioid consumption, and contribute to several complications [24]. Recently, multimodal pain management has been widely applied in TKA [25]. Multimodal pain management usually includes two or more medications with different mechanisms, such as opioids, nonsteroidal anti-inflammatory medications, steroids, and epinephrine. It is worth noting that the usage of opioids is frequently associated with side effects, such as nausea, vomiting, and pruritus [26]. Moreover, it has been reported that the pain score became worse at $24 \mathrm{~h}$ after TKA. The rebounding pain of multimodal pain management after POD 1 remains an important issue in patients who have received TKA [9]. More recently, multimodal pain management with IV acetaminophen for postoperative pain management has generated much discussion [27].
It was reported that opioid consumption was reduced from 29 to $39 \%$ in patients who received IV acetaminophen compared to a placebo in orthopedic procedures [28]. IV acetaminophen has been shown to have efficacy for reducing the consumption of opioids [15]. Murata-Ooiwa et al. [19] demonstrated that the VAS score was significantly better in the intravenous acetaminophen group than the placebo group at day 1 after TKA, with no significant differences in terms of the rate of complications between the groups. They drew the conclusion that intravenous acetaminophen provided better pain relief for patients undergoing unilateral TKA.

However, recently, other studies have reported different conclusions [18, 23]. In the O'Neal et al. study [18], the VAS scores of IV acetaminophen and placebo groups were compared, as well as total the morphine consumption, among other parameters. No significant differences were found between all groups for any outcome. Nwagbologu et al. [17] reported that the use of IV acetaminophen was not associated with a decrease in opioid use, opioid-related side effects, or any other outcomes in patients who received TKA. The current meta-analysis indicated that IV acetaminophen was associated with a statistically significant reduction in total morphine consumption by approximately $11.59 \mathrm{mg}$ compared with a control group. 


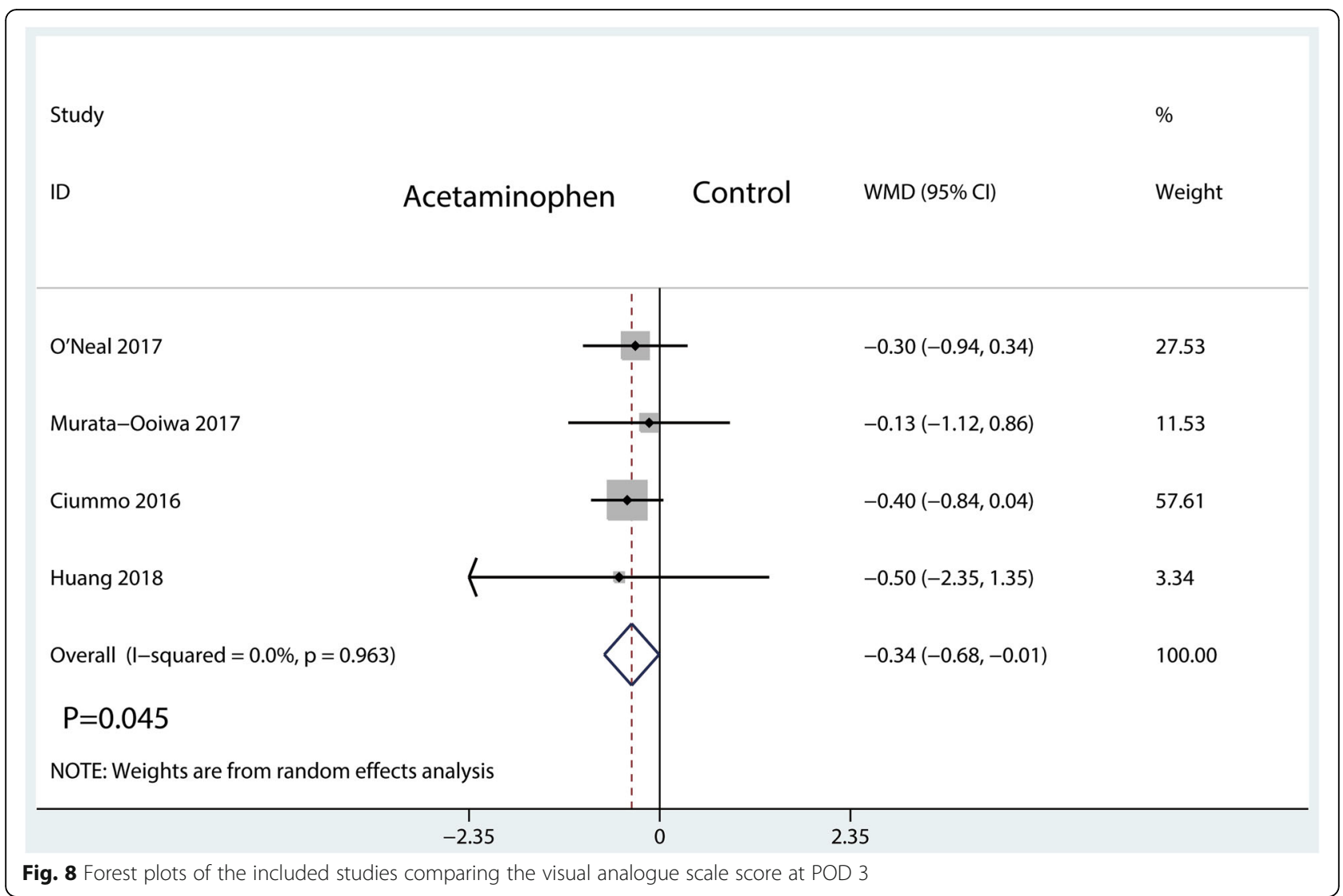

There was significantly heterogeneity between the included studies $\left(I^{2}=84.1 \%\right)$. Despite performing sensitivity analysis to diminish the impact of heterogeneity, the effect of heterogeneity still could not be eliminated completely. In the sensitivity analysis, we found that the study of Nwagbologu et al. [17] was the source of this heterogeneity. Nwagbologu et al. [17] recorded that the IV acetaminophen group and placebo group received similar doses of total morphine equivalents at 24 and $48 \mathrm{~h}$ postoperatively. We analyze the reason as follows: (1) this was a retrospective cohort study and may have had a selection bias; (2) this study comprised two doses of acetaminophen (1000 mg/day and $2000 \mathrm{mg} /$ day), so opioid-sparing effects may be related to the amount of IV acetaminophen received; (3) this study simply added onto other pain medication orders without any organized or concerted effort to use a multimodal pain regimen to reduce opioid consumption.

VAS score was also an important result in our meta-analysis. Current meta-analysis indicated that IV acetaminophen only has a beneficial role in reducing VAS score at POD 3. Murata-Ooiwa et al... [19] reported that the VAS score at 17:00 1 day after TKA was significantly better in the intravenous acetaminophen group than the placebo group. In contrast, some published studies have recently reported that IV acetaminophen has no effects on pain relief [18]. O’Neal et al. [18] reported that no significant differences were found between the IV acetaminophen and placebo groups regarding the VAS score. Similarly, Ciummo et al. [23] declared that there was no statistically significant difference in average daily postoperative VAS score.

Similar findings were reported by Murata-Ooiwa et al. [19]. In Murata-Ooiwa et al.'s study [19], there were no significant differences in the rate of complications. With regard to LOS, Ciummo et al. [23] reported that no significant differences were found. Kelly et al. [15] reported that the median length of LOS for both the IV acetaminophen and placebo groups was 3 days. In the Nwagbologu et al. study [17], the LOS in IV acetaminophen and control groups were 3.7 days and 3.9 days, respectively. These results were consistent with our meta-analysis. Therefore, we concluded that IV acetaminophen was not associated with a reduction in the length of hospital stay in patients who received TKA.

Our meta-analysis has several limitations: (1) Only six studies were included in our meta-analysis. The statistical efficacy of our results would be more reliable if more studies had been included. (2) Only English publications were included in our meta-analysis; therefore, publication bias was unavoidable. (3) Outcomes such as range of motion of the knee and knee society score were not analyzed due 
Study

$\%$

ID

Acetaminophen

Control

WMD $(95 \% \mathrm{Cl})$

Weight

Kelly 2014

Nwagbologu 2016

Ciummo 2016

Huang 2018

Overall $(I-$ squared $=58.1 \%, p=0.067)$

$P=0.226$

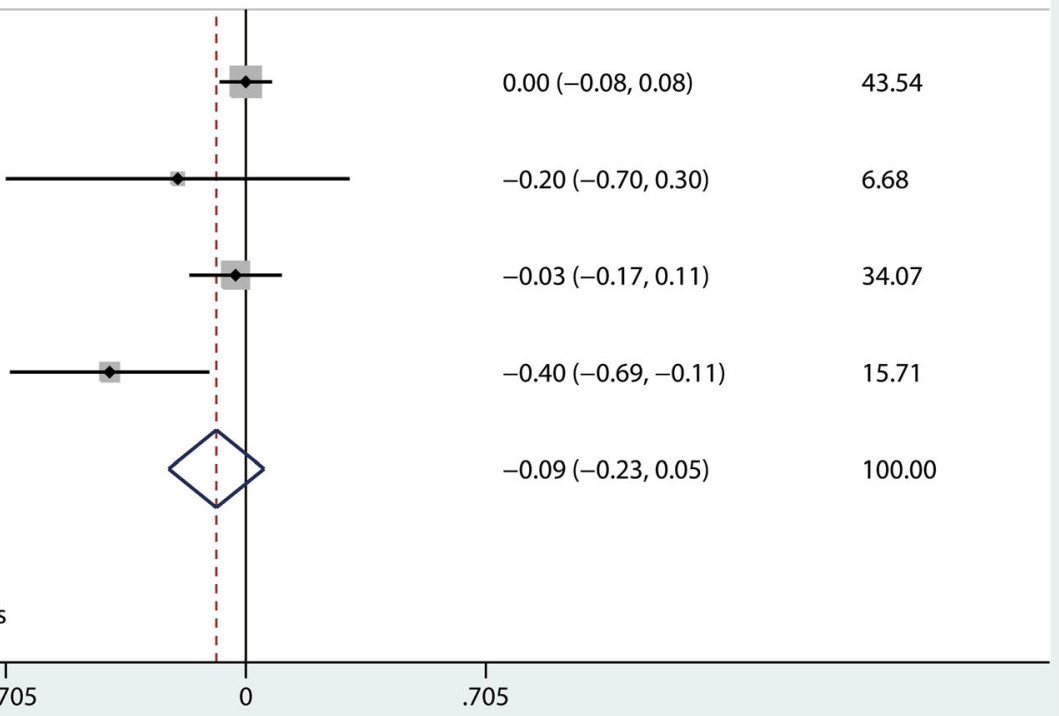

Fig. 9 Forest plots of the included studies comparing the length of hospital stay

to insufficient data. (4) Follow-ups of these studies were relatively short, and long-term follow-ups are needed to identify the knee function between these two groups. (5) There was substantial heterogeneity between included outcomes. We performed subgroup analysis and sensitivity analysis to decrease the heterogeneity; however, the overall heterogeneity was not changed after subgroup analysis or after sensitivity analysis. Thus, the results of this meta-analysis should be carefully interpreted.

\section{Conclusion}

In conclusion, based on our results, IV acetaminophen in multimodal management has shown better efficacy than a control for pain relief at POD 3 and has morphine-sparing

Table 3 Subgroup analysis of the total morphine consumption

\begin{tabular}{|c|c|c|c|c|c|}
\hline Subgroups & No. of studies & Mean difference $[95 \% \mathrm{Cl}]$ & $P$ value & $P^{2}(\%)$ & Between subgroup significance \\
\hline \multicolumn{6}{|c|}{ Total morphine consumption } \\
\hline \multicolumn{6}{|c|}{ Allocation concealment } \\
\hline Adequate & 2 & $-2.39(-43.70,38.92)$ & 0.910 & 94.5 & 0.002 \\
\hline Unclear & 2 & $-18.62(-23.56,-0.08)$ & 0.000 & 0 & \\
\hline \multicolumn{6}{|l|}{ Study type } \\
\hline RCT & 1 & $-19.70(-22.35,-15.43)$ & 0.029 & 0 & 0.124 \\
\hline RCS & 3 & $-20.45(-24.04,-16.85)$ & 0.038 & 0 & \\
\hline \multicolumn{6}{|c|}{ Acetaminophen dose } \\
\hline 1000 mg/day & 3 & $-4.42(-33.04,24.20)$ & 0.762 & 89.4 & 0.139 \\
\hline 4000 mg/day & 1 & $-19.00(-24.03,-13.97)$ & 0.000 & & \\
\hline \multicolumn{6}{|l|}{ Anesthesia } \\
\hline GA & 2 & $-2.39(-43.70,38.92)$ & 0.910 & 94.5 & 0.048 \\
\hline SA & 2 & $-18.62(-23.56,-13.68)$ & 0.000 & & \\
\hline
\end{tabular}

GA general anesthesia, $S A$ spinal anesthesia, $R C T$ randomized controlled trials, $R C S$ retrospective controlled studies 
effects. We identified six studies; in the future, the multimodal pain management protocol after TKA may change when more studies are published and included in the meta-analysis. Due to the limited studies and participants, further high-quality studies with more patients are needed to validate the optimal dose of IV-acetaminophen.

\section{Additional file}

Additional file 1: Figure S1. Sensitivity analysis of the total morphine equivalent consumption (A), morphine equivalent consumption at POD 1 (B), visual analogue scale score at POD 1 (C), visual analogue scale score at POD 2 (D), visual analogue scale score at POD 3 (E), and length of hospital stay (F). (TIF 860 kb)

\section{Abbreviations}

Cl: Confidence interval; IV: Intravenous; NOS: Newcastle-Ottawa Quality Assessment Scale; OA: Osteoarthritis; POD: Postoperative day; RA: Rheumatoid arthritis; RCS: Retrospective cohort studies; RCTs: Randomized controlled trials; RR: Risk ratio; TKA: Total knee arthroplasty; VAS: Visual analogue scale; WMD: Weighted mean difference

\section{Funding}

There is no funding for this article.

\section{Availability of data and materials}

We state that the data will not be shared since all of the raw data are present in the figures included in the article.

\section{Consent for publication}

Not applicable.

\section{Authors' contributions}

SBS and XBW conceived the study design. JMS performed the study, collected the data, and contributed to the study design. SFG and ZXC prepared the manuscript. YW edited the manuscript. All authors read and approved the final manuscript

\section{Ethics approval and consent to participate}

None

\section{Competing interests}

The authors declare that they have no competing interests.

\section{Publisher's Note}

Springer Nature remains neutral with regard to jurisdictional claims in published maps and institutional affiliations.

\section{Received: 6 July 2018 Accepted: 19 September 2018} Published online: 11 October 2018

\section{References}

1. Sun X, Su Z. A meta-analysis of unicompartmental knee arthroplasty revised to total knee arthroplasty versus primary total knee arthroplasty. J Orthop Surg Res. 2018;13(1):158.

2. Dong P, Tang X, Cheng R, Wang J. Comparison of the efficacy of different analgesia treatments for total knee arthroplasty: a network meta-analysis. Clin J Pain. 2018;34(11):1047-60

3. Li C, Qu J, Pan S, Qu Y. Local infiltration anesthesia versus epidural analgesia for postoperative pain control in total knee arthroplasty: a systematic review and meta-analysis. J Orthop Surg Res. 2018;13(1):112.

4. Mont MA, Beaver WB, Dysart SH, Barrington JW, Del Gaizo DJ. Local infiltration analgesia with liposomal bupivacaine improves pain scores and reduces opioid use after total knee arthroplasty: results of a randomized controlled trial. J Arthroplast. 2018;33(1):90-6.

5. Suarez JC, Al-Mansoori AA, Kanwar S, Semien GA, Villa JM, McNamara CA, Patel PD. Effectiveness of novel adjuncts in pain management following total knee arthroplasty: a randomized clinical trial. J Arthroplast. 2018;33(7s): S136-s141.

6. Gaffney CJ, Pelt CE, Gililland JM, Peters CL. Perioperative pain management in hip and knee arthroplasty. Orthop clin North Am. 2017; 48(4):407-19.

7. Brooks E, Freter SH, Bowles SK, Amirault D. Multimodal pain management in older elective arthroplasty patients. Geriatric orthop surg rehabilitation. 2017;8(3):151-4

8. Tsukada S, Wakui M, Hoshino A. Postoperative epidural analgesia compared with intraoperative periarticular injection for pain control following total knee arthroplasty under spinal anesthesia: a randomized controlled trial. J Bone Joint Surg Am. 2014;96(17):1433-8.

9. Tsukada S, Wakui M, Hoshino A. The impact of including corticosteroid in a periarticular injection for pain control after total knee arthroplasty: a double-blind randomised controlled trial. bone joint j. 2016;98-b(2):194-200.

10. Dahl JB, Rosenberg J, Dirkes WE, Mogensen T, Kehlet H. Prevention of postoperative pain by balanced analgesia. Br J Anaesth. 1990;64(4): 518-20.

11. Cancienne JM, Patel KJ, Browne JA, Werner BC. Narcotic use and total knee arthroplasty. J Arthroplast. 2018;33(1):113-8.

12. Saurabh S, Smith JK, Pedersen M, Jose P, Nau P, Samuel I. Scheduled intravenous acetaminophen reduces postoperative narcotic analgesic demand and requirement after laparoscopic roux-en-Y gastric bypass. Surg Obes Relat Dis. 2015;11(2):424-30.

13. Mont MA, Lovelace B, Pham AT, Hansen RN, Chughtai M, Gwam CU, Khlopas A, Barrington JW. Intravenous acetaminophen may be associated with reduced odds of 30-day readmission after total knee arthroplasty. j knee surg. 2018.

14. Subramanyam R, Varughese A, Kurth CD, Eckman MH. Cost-effectiveness of intravenous acetaminophen for pediatric tonsillectomy. Paediatr Anaesth. 2014:24(5):467-75.

15. Kelly JS, Opsha Y, Costello J, Schiller D, Hola ET. Opioid use in knee arthroplasty after receiving intravenous acetaminophen. Pharmacotherapy. 2014;34(Suppl 1):22s-6s.

16. Blank JJ, Berger NG, Dux JP, Ali F, Ludwig KA, Peterson CY. The impact of intravenous acetaminophen on pain after abdominal surgery: a metaanalysis. J Surg Res. 2018:227:234-45.

17. Nwagbologu N, Sarangarm P, D'Angio R. Effect of intravenous acetaminophen on postoperative opioid consumption in adult orthopedic surgery patients. Hosp Pharm. 2016;51(9):730-7.

18. O'Neal JB, Freiberg AA, Yelle MD, Jiang Y, Zhang C, Gu Y, Kong X, Jian W, O'Neal WT, Wang J. Intravenous vs oral acetaminophen as an adjunct to multimodal analgesia after total knee arthroplasty: a prospective, randomized, double-blind clinical trial. J Arthroplast. 2017; 32(10):3029-33.

19. Murata-Ooiwa M, Tsukada S, Wakui M. Intravenous acetaminophen in multimodal pain management for patients undergoing total knee arthroplasty: a randomized, double-blind, placebo-controlled trial. J Arthroplast. 2017;32(10):3024-8

20. Apfel C, Jahr JR, Kelly CL, Ang RY, Oderda GM. Effect of i.v. acetaminophen on total hip or knee replacement surgery: a case-matched evaluation of a national patient database. Am J Health Syst Pharm. 2015:72(22):1961-8.

21. Huang PS, Gleason SM, Shah JA, Buros AF, Hoffman DA. Efficacy of intravenous acetaminophen for postoperative analgesia in primary total knee arthroplasty. J Arthroplast. 2018;33(4):1052-6.

22. Hartling L, Ospina M, Liang Y, Dryden DM, Hooton N, Krebs Seida J, Klassen TP. Risk of bias versus quality assessment of randomised controlled trials: cross sectional study. BMJ (Clinical research ed). 2009;339:b4012.

23. Ciummo F, Cheon E, Samide J, Habib H, Abraham T, Tischler H. 1570: multimodal pain management in total knee replacement with or without intravenous acetaminophen. Crit Care Med. 2016:44(12):468

24. Laoruengthana A, Rattanaprichavej P, Rasamimongkol S, Galassi M. Anterior vs posterior periarticular multimodal drug injections: a randomized, controlled trial in simultaneous bilateral total knee arthroplasty. J Arthroplast. 2017:32(7):2100-4.

25. Parvizi J, Miller AG, Gandhi K. Multimodal pain management after total joint arthroplasty. J Bone Joint Surg Am. 2011:93(11):1075-84.

26. Jiang J, Teng Y, Fan Z, Khan MS, Cui Z, Xia Y. The efficacy of periarticular multimodal drug injection for postoperative pain management in total knee or hip arthroplasty. J Arthroplast. 2013;28(10):1882-7. 
27. Politi JR, Davis RL 2nd, Matrka AK. Randomized prospective trial comparing the use of intravenous versus oral acetaminophen in total joint arthroplasty. J Arthroplast. 2017;32(4):1125-7.

28. Sinatra RS, Jahr JS, Reynolds LW, Viscusi ER, Groudine SB, Payen-Champenois C. Efficacy and safety of single and repeated administration of $1 \mathrm{gram}$ intravenous acetaminophen injection (paracetamol) for pain management after major orthopedic surgery. Anesthesiology. 2005;102(4):822-31.

Ready to submit your research? Choose BMC and benefit from:

- fast, convenient online submission

- thorough peer review by experienced researchers in your field

- rapid publication on acceptance

- support for research data, including large and complex data types

- gold Open Access which fosters wider collaboration and increased citations

- maximum visibility for your research: over $100 \mathrm{M}$ website views per year

At $\mathrm{BMC}$, research is always in progress.

Learn more biomedcentral.com/submissions 